\title{
Evaluation of Two Types of Integrals Using Maple
}

\author{
Chii-Huei Yu \\ Department of Management and Information, Nan Jeon University of Science and Technology, Tainan City, 73746, Taiwan \\ *Corresponding Author: chiihuei@mail.njtc.edu.tw
}

Copyright $@ 2014$ Horizon Research Publishing All rights reserved.

\begin{abstract}
This paper uses the mathematical software Maple for the auxiliary tool to study two types of integrals. We can obtain the infinite series forms of these two types of integrals by using binomial series and integration term by term theorem. On the other hand, we provide some examples to do calculation practically. The research methods adopted in this study involved finding solutions through manual calculations and verifying these solutions by using Maple. This type of research method not only allows the discovery of calculation errors, but also helps modify the original directions of thinking from manual and Maple calculations. For this reason, Maple provides insights and guidance regarding problem-solving methods.
\end{abstract}

Keywords Integrals, Infinite Series Forms, Binomial Series, Integration Term By Term Theorem, Maple

\section{Introduction}

The computer algebra system (CAS) has been widely employed in mathematical and scientific studies. The rapid computations and the visually appealing graphical interface of the program render creative research possible. Maple possesses significance among mathematical calculation systems and can be considered a leading tool in the CAS field. The superiority of Maple lies in its simple instructions and ease of use, which enable beginners to learn the operating techniques in a short period. In addition, through the numerical and symbolic computations performed by Maple, the logic of thinking can be converted into a series of instructions. The computation results of Maple can be used to modify our previous thinking directions, thereby forming direct and constructive feedback that can aid in improving understanding of problems and cultivating research interests. Inquiring through an online support system provided by Maple or browsing the Maple website (www.maplesoft.com) can facilitate further understanding of Maple and might provide unexpected insights. As for the instructions and operations of Maple, we can refer to [1-7].

In calculus and engineering mathematics courses, we learnt many methods to solve the integral problems, including change of variables method, integration by parts method, partial fractions method, trigonometric substitution method, and so on. In this paper, we study the following two types of integrals which are not easy to obtain their answers using the methods mentioned above.

$$
\begin{aligned}
& \int e^{a x} \cosh ^{r}(b x+c) d x \\
& \int e^{a x} \sinh ^{r}(b x+c) d x
\end{aligned}
$$

Where $a, b, c, r$ are real numbers, and $b \neq 0$. We can obtain the infinite series forms of these two types of integrals by using binomial series and integration term by term theorem ; these are the major results of this study (i.e., Theorems 1 and 2). As for the study of related integral problems can refer to [8-22]. On the other hand, we propose some examples to do calculation practically. The research methods adopted in this study involved finding solutions through manual calculations and verifying these solutions by using Maple. This type of research method not only allows the discovery of calculation errors, but also helps modify the original directions of thinking from manual and Maple calculations. Therefore, Maple provides insights and guidance regarding problem-solving methods.

\section{Main Results}

Firstly, we introduce a notation and two important theorems used in this study.

\subsection{Notation}

If $r$ is a real number, $k$ is a positive integer. We define $(r)_{k}=r(r-1) \cdots(r-k+1)$, and $(r)_{0}=1$.

\subsection{Binomial series}

$(1+u)^{r}=\sum_{k=0}^{\infty} \frac{(r)_{k}}{k !} u^{k}$, where $u, r$ are real numbers, $|u|<1$

\subsection{Integration term by term theorem ([23])}


Suppose $\left\{g_{n}\right\}_{n=0}^{\infty}$ is a sequence of Lebesgue integrable functions defined on an inteval $I$. If $\sum_{n=0}^{\infty} \int_{I}\left|g_{n}\right|$ is convergent, then $\int_{I} \sum_{n=0}^{\infty} g_{n}=\sum_{n=0}^{\infty} \int_{I} g_{n}$.

The following is the first result in this study, we determine the infinite series form of the integral (1).

2.4. Theorem 1 Suppose $a, b, c, r$ are real numbers, $b \neq 0$, and $C$ is a constant.

Case (A): If $b x+c>0$, and $\frac{a+b r}{2 b}$ is not a non-negative integer, then the integral

$$
\begin{gathered}
\int e^{a x} \cosh ^{r}(b x+c) d x \\
=2^{-r} e^{(a+b r) x+c r} \sum_{k=0}^{\infty} \frac{(r)_{k}}{k !(-2 b k+a+b r)} \cdot e^{-2(b x+c) k}+C
\end{gathered}
$$

Case (B): If $b x+c>0$, and $k_{0}=\frac{a+b r}{2 b}$ is a non-negative integer, then the integral

$$
\begin{gathered}
\int e^{a x} \cosh ^{r}(b x+c) d x \\
=2^{-r} e^{(a+b r) x+c r} \sum_{\substack{k=0 \\
k \neq k_{0}}}^{\infty} \frac{(r)_{k}}{k !(-2 b k+a+b r)} \cdot e^{-2(b x+c) k} \\
+\frac{2^{-r}(r)_{k_{0}} e^{\left(c r-2 c k_{0}\right)}}{k_{0} !} \cdot x+C
\end{gathered}
$$

Case (C): If $b x+c<0$, and $\frac{b r-a}{2 b}$ is not a non-negative integer, then

$$
\begin{gathered}
\int e^{a x} \cosh ^{r}(b x+c) d x \\
=2^{-r} e^{(a-b r) x-c r} \sum_{k=0}^{\infty} \frac{(r)_{k}}{k !(2 b k+a-b r)} \cdot e^{2(b x+c) k}+C(5)
\end{gathered}
$$

Case (D): If $b x+c<0$, and $k_{1}=\frac{b r-a}{2 b}$ is a non-negative integer, then

$$
\begin{array}{r}
\int e^{a x} \cosh ^{r}(b x+c) d x \\
=2^{-r} e^{(a-b r) x-c r} \sum_{\substack{k=0 \\
k \neq k 1}}^{\infty} \frac{(r)_{k}}{k !(2 b k+a-b r)} \cdot e^{2(b x+c) k} \\
+\frac{2^{-r}(r)_{k 1} e^{\left(-c r+2 c k_{1}\right)}}{k_{1} !} \cdot x+C
\end{array}
$$

\subsubsection{Proof}

Case (A): If $b x+c>0$, and $\frac{a+b r}{2 b}$ is not a non-negative integer. Because

$$
\begin{gathered}
e^{a x} \cosh ^{r}(b x+c) \\
=e^{a x}\left[\frac{1}{2}\left(e^{b x+c}+e^{-(b x+c)}\right)\right]^{r} \\
=2^{-r} e^{a x+r(b x+c)} \cdot\left[1+e^{-2(b x+c)}\right]^{r} \\
=2^{-r} e^{a x+r(b x+c)} \sum_{k=0}^{\infty} \frac{(r)_{k}}{k !} e^{-2(b x+c) k}
\end{gathered}
$$

(By binomial series)

$$
=2^{-r} e^{c r} \sum_{k=0}^{\infty} \frac{(r)_{k} \cdot e^{-2 c k}}{k !} e^{(-2 b k+a+b r) x}
$$

Therefore,

$$
\begin{aligned}
& \int e^{a x} \cosh ^{r}(b x+c) d x \\
& =2^{-r} e^{c r} \int \sum_{k=0}^{\infty} \frac{(r)_{k} \cdot e^{-2 c k}}{k !} e^{(-2 b k+a+b r) x} d x \\
& =2^{-r} e^{c r} \sum_{k=0}^{\infty} \frac{(r)_{k} \cdot e^{-2 c k}}{k !} \int e^{(-2 b k+a+b r) x} d x \\
& =2^{-r} e^{c r} \sum_{k=0}^{\infty} \frac{(r)_{k} \cdot e^{-2 c k}}{k !(-2 b k+a+b r)} \cdot e^{(-2 b k+a+b r) x}+C \\
& =2^{-r} e^{(a+b r) x+c r} \sum_{k=0}^{\infty} \frac{(r)_{k}}{k !(-2 b k+a+b r)} \cdot e^{-2(b x+c) k}+C \\
& e^{a x} \cosh ^{r}(b x+c) \\
& =2^{-r} e^{c r} \sum_{\substack{k=0 \\
k \neq k_{0}}}^{\infty} \frac{(r)_{k} \cdot e^{-2 c k}}{k !} e^{(-2 b k+a+b r) x} \\
& +\frac{2^{-r}(r)_{k_{0}} e^{\left(c r-2 c k_{0}\right)}}{k_{0} !}
\end{aligned}
$$

Thus, the integral

$$
\int e^{a x} \cosh ^{r}(b x+c) d x
$$




$$
\begin{gathered}
=2^{-r} e^{(a+b r) x+c r} \sum_{\substack{k=0 \\
k \neq k_{0}}}^{\infty} \frac{(r)_{k}}{k !(-2 b k+a+b r)} \cdot e^{-2(b x+c) k} \\
+\frac{2^{-r}(r)_{k_{0}} e^{\left(c r-2 c k_{0}\right)}}{k_{0} !} \cdot x+C
\end{gathered}
$$

Case (C): If $b x+c<0$, and $\frac{b r-a}{2 b}$ is not a non-negative integer. Because

$$
\begin{array}{r}
e^{a x} \cosh ^{r}(b x+c) \\
=e^{a x} \cosh ^{r}[-(b x+c)] \\
=2^{-r} e^{-c r} \sum_{k=0}^{\infty} \frac{(r)_{k} \cdot e^{2 c k}}{k !} e^{(2 b k+a-b r) x}
\end{array}
$$

(By (7))

Hence, by integration term by term theorem, the integral

$$
\begin{array}{r}
\int e^{a x} \cosh ^{r}(b x+c) d x \\
=2^{-r} e^{-c r} \sum_{k=0}^{\infty} \frac{(r)_{k} e^{2 c k}}{k !} \cdot \int e^{(2 b k+a-b r) x} d x \\
=2^{-r} e^{(a-b r) x-c r} \sum_{k=0}^{\infty} \frac{(r)_{k}}{k !(2 b k+a-b r)} \cdot e^{2(b x+c) k}+C
\end{array}
$$

Case (D). If $b x+c<0$, and $k_{1}=\frac{b r-a}{2 b}$ is a non-negative integer. Then by (9), we have

$$
\begin{aligned}
e^{a x} \cosh ^{r}(b x+c) \\
=2^{-r} e^{-c r} \sum_{\substack{k=0 \\
k \neq k_{1}}}^{\infty} \frac{(r)_{k} \cdot e^{2 c k}}{k !} e^{(2 b k+a-b r) x} \\
+\frac{2^{-r}(r)_{k_{1}} e^{\left(-c r+2 c k_{1}\right)}}{k_{1} !}
\end{aligned}
$$

Thus, using integration term by term theorem, the integral

$$
\begin{array}{r}
\int e^{a x} \cosh ^{r}(b x+c) d x \\
=2^{-r} e^{(a-b r) x-c r} \sum_{\substack{k=0 \\
k \neq k 1}}^{\infty} \frac{(r)_{k}}{k !(2 b k+a-b r)} \cdot e^{2(b x+c) k} \\
+\frac{2^{-r}(r)_{k 1} e^{\left(-c r+2 c k_{1}\right)}}{k_{1} !} \cdot x+C
\end{array}
$$

The following is the second major result in this paper, we determine the infinite series form of the integral (2).

\subsection{Theorem 2}

Let the assumptions be the same as Theorem 1.

Case (A): If $b x+c>0$, and $\frac{a+b r}{2 b}$ is not a non-negative integer, then the integral

$$
\begin{gathered}
\int e^{a x} \sinh ^{r}(b x+c) d x \\
=2^{-r} e^{(a+b r) x+c r} \sum_{k=0}^{\infty} \frac{(-1)^{k}(r)_{k}}{k !(-2 b k+a+b r)} \cdot e^{-2(b x+c) k}+C
\end{gathered}
$$

Case (B): If $b x+c>0$, and $k_{0}=\frac{a+b r}{2 b}$ is a non-negative integer, then

$$
\begin{gathered}
\int e^{a x} \sinh ^{r}(b x+c) d x \\
=2^{-r} e^{(a+b r) x+c r} \sum_{\substack{k=0 \\
k \neq k_{0}}}^{\infty} \frac{(-1)^{k}(r)_{k}}{k !(-2 b k+a+b r)} \cdot e^{-2(b x+c) k} \\
+\frac{2^{-r}(-1)^{k_{0}}(r)_{k_{0}} e^{\left(c r-2 c k_{0}\right)}}{k_{0} !} \cdot x+C
\end{gathered}
$$

Case (C): If $b x+c<0, \frac{b r-a}{2 b}$ is not a non-negative integer, and $(-1)^{r}$ exists. Then

$$
\begin{gathered}
\int e^{a x} \sinh ^{r}(b x+c) d x \\
=(-1)^{r} 2^{-r} e^{(a-b r) x-c r} \sum_{k=0}^{\infty} \frac{(-1)^{k}(r)_{k}}{k !(2 b k+a-b r)} \cdot e^{2(b x+c) k}+C(13)
\end{gathered}
$$

Case (D): If $b x+c<0, k_{1}=\frac{b r-a}{2 b}$ is a non-negative integer, and $(-1)^{r}$ exists. Then

$$
\begin{gathered}
\int e^{a x} \sinh ^{r}(b x+c) d x \\
=(-1)^{r} 2^{-r} e^{(a-b r) x-c r} \sum_{\substack{k=0 \\
k \neq k 1}}^{\infty} \frac{(-1)^{k}(r)_{k}}{k !(2 b k+a-b r)} \cdot e^{2(b x+c) k} \\
+\frac{(-1)^{k_{1}+r} 2^{-r}(r)_{k 1} e^{\left(-c r+2 c k_{1}\right)}}{k_{1} !} \cdot x+C
\end{gathered}
$$

\subsubsection{Proof}

Case (A): If $b x+c>0$, and $\frac{a+b r}{2 b}$ is not a non-negative integer. Because

$$
e^{a x} \sinh ^{r}(b x+c)
$$




$$
\begin{gathered}
=e^{a x}\left[\frac{1}{2}\left(e^{b x+c}-e^{-(b x+c)}\right)\right]^{r} \\
=2^{-r} e^{a x+r(b x+c)} \cdot\left[1-e^{-2(b x+c)}\right]^{r} \\
=2^{-r} e^{a x+r(b x+c)} \sum_{k=0}^{\infty} \frac{(-1)^{k}(r)_{k}}{k !} e^{-2(b x+c) k}
\end{gathered}
$$

(By binomial series)

$$
=2^{-r} e^{c r} \sum_{k=0}^{\infty} \frac{(-1)^{k}(r)_{k} \cdot e^{-2 c k}}{k !} e^{(-2 b k+a+b r) x}
$$

Therefore,

$$
\begin{gathered}
\int e^{a x} \sinh ^{r}(b x+c) d x \\
=2^{-r} e^{c r} \sum_{k=0}^{\infty} \frac{(-1)^{k}(r)_{k} \cdot e^{-2 c k}}{k !} \int e^{(-2 b k+a+b r) x} d x
\end{gathered}
$$

(By integration term by term theorem)

$$
=2^{-r} e^{(a+b r) x+c r} \sum_{k=0}^{\infty} \frac{(-1)^{k}(r)_{k}}{k !(-2 b k+a+b r)} \cdot e^{-2(b x+c) k}+C
$$

Case (B): If $b x+c>0$, and $k_{\mathrm{O}}=\frac{a+b r}{2 b}$ is a non-negative integer. Then by (15), we obtain

$$
\begin{gathered}
e^{a x} \sinh ^{r}(b x+c) \\
=2^{-r} e^{c r} \sum_{\substack{k=0 \\
k \neq k_{0}}}^{\infty} \frac{(-1)^{k}(r)_{k} \cdot e^{-2 c k}}{k !} e^{(-2 b k+a+b r) x} \\
+\frac{2^{-r}(-1)^{k_{0}}(r)_{k_{0}} e^{\left(c r-2 c k_{0}\right)}}{k_{0} !}
\end{gathered}
$$

Thus, the integral

$$
\begin{gathered}
\int e^{a x} \sinh ^{r}(b x+c) d x \\
=2^{-r} e^{(a+b r) x+c r} \sum_{\substack{k=0 \\
k \neq k_{0}}}^{\infty} \frac{(-1)^{k}(r)_{k}}{k !(-2 b k+a+b r)} \cdot e^{-2(b x+c) k} \\
+\frac{2^{-r}(-1)^{k_{0}}(r)_{k_{0}} e^{\left(c r-2 c k_{0}\right)}}{k_{0} !} \cdot x+C
\end{gathered}
$$

Case (C): If $b x+c<0$, and $\frac{b r-a}{2 b}$ is not a non-negative integer, and $(-1)^{r}$ exists. Because

$$
\begin{gathered}
e^{a x} \sinh ^{r}(b x+c) \\
=(-1)^{r} e^{a x} \sinh ^{r}[-(b x+c)]
\end{gathered}
$$

$$
=(-1)^{r} 2^{-r} e^{-c r} \sum_{k=0}^{\infty} \frac{(-1)^{k}(r)_{k} \cdot e^{2 c k}}{k !} e^{(2 b k+a-b r) x}
$$

Hence, by integration term by term theorem, the integral

$$
\begin{gathered}
\int e^{a x} \sinh ^{r}(b x+c) d x \\
=(-1)^{r} 2^{-r} e^{-c r} \sum_{k=0}^{\infty} \frac{(-1)^{k}(r)_{k} \cdot e^{2 c k}}{k !} \cdot \int e^{(2 b k+a-b r) x} d x \\
=(-1)^{r} 2^{-r} e^{(a-b r) x-c r} \sum_{k=0}^{\infty} \frac{(-1)^{k}(r)_{k}}{k !(2 b k+a-b r)} \cdot e^{2(b x+c) k}+C
\end{gathered}
$$

Case (D). If $b x+c<0, k_{1}=\frac{b r-a}{2 b}$ is a non-negative integer, and $(-1)^{r}$ exists. Then by (17), we have

$$
\begin{aligned}
& =(-1)^{r} 2^{-r} e^{-c r} \sum_{\substack{k=0 \\
k \neq k_{1}}}^{\infty} \frac{(-1)^{k}(r)_{k} \cdot e^{2 c k}}{k !} e^{(2 b k+a-b r) x} \\
& +\frac{(-1)^{k_{1}+r} 2^{-r}(r)_{k_{1}} e^{\left(-c r+2 c k_{1}\right)}}{k_{1} !} \\
& \text { Thus, using integration term by term theorem, the integral } \\
& =(-1)^{r} 2^{-r} e^{(a-b r) x-c r} \sum_{\substack{k=0 \\
k \neq k 1}}^{\infty} \frac{(-1)^{k}(r)_{k}}{k !(2 b k+a-b r)} \cdot e^{2(b x+c) k} \sinh ^{r}(b x+c) d x \\
& +\frac{\left.(-1)^{k_{1}+r} 2^{-r}(r)_{k 1} e^{(-c r+2 c k}\right)}{k_{1} !} \cdot x+C
\end{aligned}
$$

\section{Examples}

In the following, for the two types of integrals in this study, we provide some examples and use Theorems 1, 2 to determine their infinite series forms. On the other hand, we evaluate some related definite integrals and employ Maple to calculate the approximations of these definite integrals and their solutions for verifying our answers.

\subsection{Example 1}

In Theorem 1, let $a=6, b=2, c=5, r=1 / 2$.

3.1.1. If $2 x+5>0$, then by Case (A) of Theorem 1, we obtain the integral 


$$
\begin{gathered}
\int e^{6 x} \sqrt{\cosh (2 x+5)} d x \\
=\frac{1}{\sqrt{2}} e^{7 x+5 / 2} \sum_{k=0}^{\infty} \frac{(1 / 2)_{k}}{k !(-4 k+7)} \cdot e^{-2(2 x+5) k}+C
\end{gathered}
$$

Thus, we can determine the following definite integral

$$
\begin{gathered}
\int_{-1}^{3} e^{6 x} \sqrt{\cosh (2 x+5)} d x \\
=\frac{1}{\sqrt{2}} e^{47 / 2} \sum_{k=0}^{\infty} \frac{(1 / 2)_{k}}{k !(-4 k+7)} \cdot e^{-22 k} \\
-\frac{1}{\sqrt{2}} e^{-9 / 2} \sum_{k=0}^{\infty} \frac{(1 / 2)_{k}}{k !(-4 k+7)} \cdot e^{-6 k}
\end{gathered}
$$

Next, we use Maple to verify the correctness of (20).

$>\operatorname{evalf}\left(\operatorname{int}\left(\exp \left(6^{*} \mathrm{x}\right) *(\cosh (2 * \mathrm{x}+5))^{\wedge}(1 / 2), \mathrm{x}=-1 . .3\right), 22\right)$;

$$
\begin{aligned}
& 1.622958022476558747900 \cdot 10^{9} \\
- & 1.976915606321369307509 \cdot 10^{-11} \mathrm{I}
\end{aligned}
$$

$>\operatorname{evalf}(1 / \operatorname{sqrt}(2) * \exp (47 / 2) * \operatorname{sum}(\operatorname{product}(1 / 2-\mathrm{j}, \mathrm{j}=0 . .(\mathrm{k}-1)) /(\mathrm{k}$ $! *(-4 * \mathrm{k}+7)) * \exp (-22 * \mathrm{k}), \mathrm{k}=0$..infinity $)-1 / \mathrm{sqrt}(2) * \exp (-9 / 2) * \mathrm{~s}$ um(product $(1 / 2-\mathrm{j}, \mathrm{j}=0 . .(\mathrm{k}-1)) /(\mathrm{k} ! *(-4 * \mathrm{k}+7)) * \exp (-6 * \mathrm{k}), \mathrm{k}=0$. infinity),22);

\section{$1.622958022476558747899 \cdot 10^{9}$}

The above answer obtained by Maple appears I $(=\sqrt{-1})$, it is because Maple calculates by using special functions built in. The imaginary part is very small, so can be ignored.

3.1.2. If $2 x+5<0$, then by Case (C) of Theorem 1 , we obtain

$$
\begin{gathered}
\int e^{6 x} \sqrt{\cosh (2 x+5)} d x \\
=\frac{1}{\sqrt{2}} e^{5 x-5 / 2} \sum_{k=0}^{\infty} \frac{(1 / 2)_{k}}{k !(4 k+5)} \cdot e^{2(2 x+5) k}+C
\end{gathered}
$$

Hence, the definite integral

$$
\begin{gathered}
\int_{-4}^{-3} e^{6 x} \sqrt{\cosh (2 x+5)} d x \\
=\frac{1}{\sqrt{2}} e^{-35 / 2} \sum_{k=0}^{\infty} \frac{(1 / 2)_{k}}{k !(4 k+5)} \cdot e^{-2 k} \\
-\frac{1}{\sqrt{2}} e^{-45 / 2} \sum_{k=0}^{\infty} \frac{(1 / 2)_{k}}{k !(4 k+5)} \cdot e^{-6 k}
\end{gathered}
$$

Using Maple to verify the correctness of (22) as follows: $>\operatorname{evalf}\left(\operatorname{int}\left(\exp \left(6^{*} \mathrm{x}\right) *(\cosh (2 * \mathrm{x}+5))^{\wedge}(1 / 2), \mathrm{x}=-4 . .-3\right), 45\right)$;
$+1.219086262572477058348400030755666207 \cdot 10^{-42} \mathrm{I}$ $>\operatorname{evalf}(1 / \operatorname{sqrt}(2) * \exp (-35 / 2) * \operatorname{sum}(\operatorname{product}(1 / 2-\mathrm{j}, \mathrm{j}=0 . .(\mathrm{k}-1)) /($ $\mathrm{k} ! *(4 * \mathrm{k}+5)) * \exp (-2 * \mathrm{k}), \mathrm{k}=0$..infinity $)-1 / \operatorname{sqrt}(2) * \exp (-45 / 2)^{*}$ $\operatorname{sum}(\operatorname{product}(1 / 2-\mathrm{j}, \mathrm{j}=0 . .(\mathrm{k}-1)) /(\mathrm{k} ! *(4 * \mathrm{k}+5)) * \exp (-6 * \mathrm{k}), \mathrm{k}=0$.. infinity),45);

\section{$3.657666703983875966261555867721949 \cdot 10^{-9}$}

The imaginary part of the above answer obtained by Maple is very small, so can be ignored.

\subsection{Example 2}

In Theorem 1, taking $a=4, b=2, c=-4$, $r=8$.

3.2.1. If $2 x-4>0$, by Case (B) of Theorem 1, we obtain the integral

$$
\begin{gathered}
\int e^{4 x} \cosh ^{8}(2 x-4) d x \\
=2^{-8} e^{20 x-32} \sum_{\substack{k=0 \\
k \neq 5}}^{\infty} \frac{(8)_{k}}{k !(-4 k+20)} \cdot e^{-2(2 x-4) k} \\
+\frac{2^{-8}(8)_{5} e^{8}}{5 !} \cdot x+C \\
=2^{-8} e^{20 x-32} \sum_{\substack{k=0 \\
k \neq 5}}^{8} \frac{(8)_{k}}{k !(-4 k+20)} \cdot e^{-2(2 x-4) k} \\
+\frac{2^{-8}(8)_{5} e^{8}}{5 !} \cdot x+C
\end{gathered}
$$

(Because $(8)_{k}=0$ for all $k \geq 9$ )

Therefore, we can evaluate the definite integral

$$
\begin{gathered}
\int_{4}^{5} e^{4 x} \cosh ^{8}(2 x-4) d x \\
=2^{-8} e^{68} \sum_{\substack{k=0 \\
k \neq 5}}^{8} \frac{(8)_{k}}{k !(-4 k+20)} \cdot e^{-12 k} \\
-2^{-8} e^{48} \sum_{\substack{k=0 \\
k \neq 5}}^{8} \frac{(8)_{k}}{k !(-4 k+20)} \cdot e^{-8 k} \\
+\frac{2^{-8}(8)_{5} e^{8}}{5 !}
\end{gathered}
$$

We also employ Maple to verify the correctness of (24). $>$ evalf $\left(\operatorname{int}\left(\exp (4 * x) *(\cosh (2 * x-4))^{\wedge} 8, x=4 . .5\right), 28\right)$;

$$
6.649385185231551625502052849 \cdot 10^{25}
$$

$>\operatorname{evalf}\left(2^{\wedge}(-8) * \exp (68) *(\operatorname{sum}(\operatorname{product}(8-\mathrm{j}, \mathrm{j}=0 . .(\mathrm{k}-1)) /(\mathrm{k} ! *(-4\right.$ 
$* \mathrm{k}+20)) * \exp (-12 * \mathrm{k}), \mathrm{k}=0 . .4)+\operatorname{sum}(\operatorname{product}(8-\mathrm{j}, \mathrm{j}=0 . .(\mathrm{k}-1)) /(\mathrm{k}$ !*(-4*k+20))*exp(-12*k),k=6..8))-2^(-8)*exp(48)*(sum( $\operatorname{product}(8-\mathrm{j}, \mathrm{j}=0 . .(\mathrm{k}-1)) /(\mathrm{k} ! *(-4 * \mathrm{k}+20)) * \exp (-8 * \mathrm{k}), \mathrm{k}=0 . .4)+$ $\operatorname{sum}(\operatorname{product}(8-\mathrm{j}, \mathrm{j}=0 . .(\mathrm{k}-1)) /(\mathrm{k} ! *(-4 * \mathrm{k}+20)) * \exp (-8 * \mathrm{k}), \mathrm{k}=6$. $\left.8))+2^{\wedge}(-8) * \operatorname{product}(8-\mathrm{t}, \mathrm{t}=0 . .4) * \exp (8) / 5 !, 28\right)$;

\section{$6.649385185231551625502052847 \cdot 10^{25}$}

3.2.2. If $2 x-4<0$, by Case (D) of Theorem 1 , we have the integral

$$
\begin{gathered}
\int e^{4 x} \cosh ^{8}(2 x-4) d x \\
=2^{-8} e^{-12 x+32} \sum_{\substack{k=0 \\
k \neq 3}}^{8} \frac{(8)_{k}}{k !(4 k-12)} \cdot e^{2(2 x-4) k} \\
+\frac{2^{-8}(8)_{3} e^{8}}{6} \cdot x+C
\end{gathered}
$$

Thus, the definite integral

$$
\begin{gathered}
\int_{-2}^{1} e^{4 x} \cosh ^{8}(2 x-4) d x \\
=2^{-8} e^{20} \sum_{\substack{k=0 \\
k \neq 3}}^{8} \frac{(8)_{k}}{k !(4 k-12)} \cdot e^{-4 k} \\
-2^{-8} e^{56} \sum_{\substack{k=0 \\
k \neq 3}}^{8} \frac{(8)_{k}}{k !(4 k-12)} \cdot e^{-16 k} \\
+\frac{2^{-8}(8)_{3} e^{8}}{2}
\end{gathered}
$$

Verifying the correctness of (26) as follows:

$>\operatorname{evalf}(\operatorname{int}(\exp (4 * x) *(\cosh (2 * x-4)) \wedge 8, x=-2 . .1), 35)$;

$$
6.8087966166615321775945316613 \cdot 10^{20}
$$

$>\operatorname{evalf}\left(2^{\wedge}(-8) * \exp (20) *\left(\operatorname{sum}\left(\operatorname{product}(8-\mathrm{j}, \mathrm{j}=0 . .(\mathrm{k}-1)) /\left(\mathrm{k} ! *\left(4^{*}\right.\right.\right.\right.\right.$ $\mathrm{k}-12)) * \exp (-4 * \mathrm{k}), \mathrm{k}=0 . .2)+\operatorname{sum}(\operatorname{product}(8-\mathrm{j}, \mathrm{j}=0 . .(\mathrm{k}-1)) /(\mathrm{k} ! *($ $4 * \mathrm{k}-12)) * \exp (-4 * \mathrm{k}), \mathrm{k}=4 . .8))-2^{\wedge}(-8) * \exp (56) *(\operatorname{sum}($ product ( $8-\mathrm{j}, \mathrm{j}=0 . .(\mathrm{k}-1)) /(\mathrm{k} ! *(4 * \mathrm{k}-12)) * \exp (-16 * \mathrm{k}), \mathrm{k}=0 . .2)+\operatorname{sum}($ $\operatorname{product}(8-\mathrm{j}, \mathrm{j}=0 . .(\mathrm{k}-1)) /(\mathrm{k} ! *(4 * \mathrm{k}-12)) * \exp (-16 * \mathrm{k}), \mathrm{k}=4 . .8))+$ $\left.2^{\wedge}(-8) * \operatorname{product}(8-\mathrm{t}, \mathrm{t}=0 . .2) * \exp (8) / 2,35\right)$;

$$
6.8087966166615321775945316614 \cdot 10^{20}
$$

\subsection{Example 3}

In Theorem 2, let $a=-2, b=3, c=7, r=2 / 5$.

3.3.1. If $3 x+7>0$, then by Case (A) of Theorem 2, we obtain the integral

$$
\int e^{-2 x} \sinh ^{2 / 5}(3 x+7) d x
$$

$$
=2^{-2 / 5} e^{-4 x / 5+14 / 5} \sum_{k=0}^{\infty} \frac{(-1)^{k}(2 / 5)_{k}}{k !(-6 k-4 / 5)} \cdot e^{-2(3 x+7) k}+C
$$

Thus, we can evaluate the following definite integral

$$
\begin{gathered}
\int_{-2}^{4} e^{-2 x} \sinh ^{2 / 5}(3 x+7) d x \\
=2^{-2 / 5} e^{-2 / 5} \sum_{k=0}^{\infty} \frac{(-1)^{k}(2 / 5)_{k}}{k !(-6 k-4 / 5)} \cdot e^{-38 k} \\
-2^{-2 / 5} e^{22 / 5} \sum_{k=0}^{\infty} \frac{(-1)^{k}(2 / 5)_{k}}{k !(-6 k-4 / 5)} \cdot e^{-2 k}
\end{gathered}
$$

In the following, we use Maple to verify the correctness of (28).

$>\operatorname{evalf}\left(\operatorname{int}\left(\exp (-2 * \mathrm{x}) *(\sinh (3 * \mathrm{x}+7))^{\wedge}(2 / 5), \mathrm{x}=-2 . .4\right), 22\right)$;

$$
76.02268988074409315774
$$

$>\operatorname{evalf}\left(2^{\wedge}(-2 / 5)^{*} \exp (-2 / 5)^{*} \operatorname{sum}\left((-1)^{\wedge} \mathrm{k}^{*} \operatorname{product}(2 / 5-\mathrm{j}, \mathrm{j}=0 . .(\mathrm{k}\right.\right.$ $-1)) /(\mathrm{k} ! *(-6 * \mathrm{k}-4 / 5)) * \exp (-38 * \mathrm{k}), \mathrm{k}=0$..infinity $)-2^{\wedge}(-2 / 5) * \exp$ $(22 / 5) * \operatorname{sum}\left((-1)^{\wedge} \mathrm{k} * \operatorname{product}(2 / 5-\mathrm{j}, \mathrm{j}=0 . .(\mathrm{k}-1)) /(\mathrm{k} ! *(-6 * \mathrm{k}-4 / 5))\right.$ $* \exp (-2 * \mathrm{k}), \mathrm{k}=0$. .infinity $), 22)$;

\subsection{9}

3.3.2. If $3 x+7<0$, then by Case (C) of Theorem 2, we obtain

$$
\begin{gathered}
\int e^{-2 x} \sinh ^{2 / 5}(3 x+7) d x \\
=2^{-2 / 5} e^{-16 x / 5-14 / 5} \sum_{k=0}^{\infty} \frac{(-1)^{k}(2 / 5)_{k}}{k !(6 k-16 / 5)} \cdot e^{2(3 x+7) k}+C
\end{gathered}
$$

Hence, the definite integral

$$
\begin{gathered}
\int_{-6}^{-4} e^{-2 x} \sinh ^{2 / 5}(3 x+7) d x \\
=2^{-2 / 5} e^{10} \sum_{k=0}^{\infty} \frac{(-1)^{k}(2 / 5)_{k}}{k !(6 k-16 / 5)} \cdot e^{-10 k} \\
-2^{-2 / 5} e^{82 / 5} \sum_{k=0}^{\infty} \frac{(-1)^{k}(2 / 5)_{k}}{k !(6 k-16 / 5)} \cdot e^{-22 k}
\end{gathered}
$$

Using Maple to verify the correctness of (30) as follows: $>$ evalf $\left(\operatorname{int}\left(\exp (-2 * x)^{*}\left((\sinh (3 * x+7))^{\wedge} 2\right)^{\wedge}(1 / 5), x=-6 . .-4\right), 22\right)$;

$$
3.134334235315029939210 \cdot 10^{6}
$$

$>\operatorname{evalf}\left(2^{\wedge}(-2 / 5)^{*} \exp (10)^{*} \operatorname{sum}\left((-1)^{\wedge} \mathrm{k}^{*} \operatorname{product}(2 / 5-\mathrm{j}, \mathrm{j}=0 . .(\mathrm{k}-\right.\right.$ 1) $) /\left(\mathrm{k} ! *\left(6^{*} \mathrm{k}-16 / 5\right)\right)^{*} \exp \left(-10^{*} \mathrm{k}\right), \mathrm{k}=0$..infinity $)-2^{\wedge}(-2 / 5) * \exp ($ $82 / 5)^{*} \operatorname{sum}\left((-1)^{\wedge} \mathrm{k}^{*} \operatorname{product}(2 / 5-\mathrm{j}, \mathrm{j}=0 . .(\mathrm{k}-1)) /(\mathrm{k} ! *(6 * \mathrm{k}-16 / 5))\right.$ $* \exp (-22 * \mathrm{k}), \mathrm{k}=0$..infinity),22);

$$
3.134334235315029939211 \cdot 10^{6}
$$




\subsection{Example 4}

In Theorem 2, taking $a=5, b=1, c=9, r=7$.

3.4.1. If $x+9>0$, by Case (B) of Theorem 2, we obtain the integral

$$
\begin{gathered}
\int e^{5 x} \sinh ^{7}(x+9) d x \\
=2^{-7} e^{12 x+63} \sum_{\substack{k=0 \\
k \neq 6}}^{7} \frac{(-1)^{k}(7)_{k}}{k !(-2 k+12)} \cdot e^{-2(x+9) k} \\
+\frac{2^{-7}(7)_{6} e^{-45}}{6 !} \cdot x+C
\end{gathered}
$$

$$
\text { (Because }(7)_{k}=0 \text { for all } k \geq 8 \text { ) }
$$

Therefore, we can evaluate the definite integral

$$
\begin{gathered}
\int_{-6}^{2} e^{5 x} \sinh ^{7}(x+9) d x \\
=2^{-7} e^{87} \sum_{\substack{k=0 \\
k \neq 6}}^{7} \frac{(-1)^{k}(7)_{k}}{k !(-2 k+12)} \cdot e^{-22 k} \\
-2^{-7} e^{-9} \sum_{\substack{k=0 \\
k \neq 6}}^{7} \frac{(-1)^{k}(7)_{k}}{k !(-2 k+12)} \cdot e^{-6 k} \\
+\frac{2^{-7}(7)_{6} e^{-45} \cdot 8}{6 !}
\end{gathered}
$$

We also employ Maple to verify the correctness of (32). $>\operatorname{evalf}\left(\operatorname{int}\left(\exp \left(5^{*} \mathrm{x}\right) *(\sinh (\mathrm{x}+9))^{\wedge} 7, \mathrm{x}=-6 . .2\right), 28\right)$;

$$
3.955748835169142260005351598 \cdot 10^{34}
$$

$>\operatorname{evalf}\left(2^{\wedge}(-7) * \exp (87) *\left(\operatorname{sum}\left((-1)^{\wedge} \mathrm{k}^{*} \operatorname{product}(7-\mathrm{j}, \mathrm{j}=0 . .(\mathrm{k}-1)) /\right.\right.\right.$ $(\mathrm{k} ! *(-2 * \mathrm{k}+12)) * \exp (-22 * \mathrm{k}), \mathrm{k}=0 . .5)+1 / 2 * \exp (-154))-2^{\wedge}(-7)^{*}$ $\exp (-9) *\left(\operatorname{sum}\left((-1)^{\wedge} \mathrm{k} * \operatorname{product}(7-\mathrm{j}, \mathrm{j}=0 . .(\mathrm{k}-1)) /(\mathrm{k} ! *(-2 * \mathrm{k}+12))\right.\right.$ $* \exp (-6 * \mathrm{k}), \mathrm{k}=0 . .5)+1 / 2 * \exp (-42))+2^{\wedge}(-7) * \operatorname{product}(7-\mathrm{t}, \mathrm{t}=0$. $\left.5)^{*} \exp (-45) * 8 / 6 !, 28\right)$;

\section{$3.955748835169142260005351598 \cdot 10^{34}$}

3.4.2. If $x+9<0$, by Case (D) of Theorem 2, we have

$$
\begin{gathered}
\int e^{5 x} \sinh ^{7}(x+9) d x \\
=-2^{-7} e^{-2 x-63} \sum_{\substack{k=0 \\
k \neq 1}}^{7} \frac{(-1)^{k}(7)_{k}}{k !(2 k-2)} \cdot e^{2(x+9) k} \\
+2^{-7} \cdot 7 e^{-45} \cdot x+C
\end{gathered}
$$

Thus, the definite integral

$$
\begin{array}{r}
\int_{-11}^{-10} e^{5 x} \sinh ^{7}(x+9) d x \\
=-2^{-7} e^{-43} \sum_{\substack{k=0 \\
k \neq 1}}^{7} \frac{(-1)^{k}(7)_{k}}{k !(2 k-2)} \cdot e^{-2 k} \\
+2^{-7} e^{-41} \sum_{\substack{k=0 \\
k \neq 1}}^{7} \frac{(-1)^{k}(7)_{k}}{k !(2 k-2)} \cdot e^{-4 k}+2^{-7} \cdot 7 e^{-45}(34)
\end{array}
$$

Using Maple to verify the correctness of (34) as follows: $>\operatorname{evalf}\left(\operatorname{int}\left(\exp \left(5^{*} \mathrm{x}\right)^{*}(\sinh (\mathrm{x}+9))^{\wedge} 7, \mathrm{x}=-11 . .-10\right), 75\right)$;

$$
-3.9559785853641365807543518849 \cdot 10^{-21}
$$

$>\operatorname{evalf}\left(-2^{\wedge}(-7)^{*} \exp (-43)^{*}\left(-1 / 2+\operatorname{sum}\left((-1)^{\wedge} \mathrm{k}^{*} \operatorname{product}(7-\mathrm{j}, \mathrm{j}=0\right.\right.\right.$. $.(\mathrm{k}-1)) /(\mathrm{k} ! *(2 * \mathrm{k}-2)) * \exp (-2 * \mathrm{k}), \mathrm{k}=2 . .7))+2^{\wedge}(-7) * \exp (-41) *(-$ $1 / 2+\operatorname{sum}\left((-1)^{\wedge} \mathrm{k}^{*} \operatorname{product}(7-\mathrm{j}, \mathrm{j}=0 . .(\mathrm{k}-1)) /(\mathrm{k} ! *(2 * \mathrm{k}-2)) * \exp (-4\right.$ $\left.* \mathrm{k}), \mathrm{k}=2 . .7))+2^{\wedge}(-7) * 7 * \exp (-45), 35\right)$;

$$
-3.9559785853641365807543518845 \cdot 10^{-21}
$$

\section{Conclusion}

As mentioned, the binomial series and the integration term by term theorem play significant roles in the theoretical inferences of this study. In fact, the applications of these two theorems are extensive, and can be used to easily solve many difficult problems; we endeavor to conduct further studies on related applications. On the other hand, Maple also plays a vital assistive role in problem-solving. In the future, we will extend the research topic to other calculus and engineering mathematics problems and solve these problems by using Maple. These results will be used as teaching materials for Maple on education and research to enhance the connotations of calculus and engineering mathematics.

\section{REFERENCES}

[1] D. Richards, Advanced Mathematical Methods with Maple, New York: Cambridge University Press, 2002.

[2] C. Tocci and S. G. Adams, Applied Maple for Engineers and Scientists, Boston: Artech House, 1996.

[3] F. Garvan, The Maple Book, London: Chapman \& Hall/CRC, 2001.

[4] M. L. Abell and J. P. Braselton, Maple by Example, 3rd ed., New York: Elsevier Academic Press, 2005.

[5] R. J. Stroeker and J. F. Kaashoek, Discovering Mathematics with Maple : An Interactive Exploration for Mathematicians, Engineers and Econometricians, Basel: Birkhauser Verlag, 
1999.

[6] J. S. Robertson, Engineering Mathematics with Maple, New York: McGraw-Hill, 1996.

[7] C. T. J. Dodson and E. A. Gonzalez, Experiments in Mathematics Using Maple, New York: Springer-Verlag, 1995.

[8] A. A. Adams, H. Gottliebsen, S. A. Linton, and U. Martin, Automated theorem proving in support of computer algebra: symbolic definite integration as a case study, Proceedings of the 1999 International Symposium on Symbolic and Algebraic Computation, pp. 253-260, Vancouver, Canada, 1999.

[9] M. A. Nyblom, On the evaluation of a definite integral involving nested square root functions, Rocky Mountain Journal of Mathematics, Vol. 37, No. 4, pp. 1301-1304, 2007.

[10] C. Oster, Limit of a definite integral, SIAM Review, Vol. 33, No. 1, pp. 115-116, 1991.

[11] C. -H. Yu, Using Maple to study two types of integrals, International Journal of Research in Computer Applications and Robotics, Vol. 1, Issue. 4, pp. 14-22, 2013.

[12] C.-H. Yu, Application of Maple on evaluation of definite integrals, Applied Mechanics and Materials, in press.

[13] C. -H. Yu, A study on integral problems by using Maple, International Journal of Advanced Research in Computer Science and Software Engineering, Vol. 3, Issue. 7, pp. 41-46, 2013.

[14] C. -H. Yu, Evaluating some integrals with Maple, International Journal of Computer Science and Mobile Computing, Vol. 2, Issue. 7, pp. 66-71, 2013.
[15] C.-H. Yu, Application of Maple on evaluating the closed forms of two types of integrals, Proceedings of the 17th Mobile Computing Workshop, ID16, 2012.

[16] C.-H. Yu, Application of Maple on some integral problems, Proceedings of the International Conference on Safety \& Security Management and Engineering Technology 2012, pp. 290-294, 2012.

[17] C.-H. Yu, Application of Maple on the integral problem of some type of rational functions, Proceedings of the Annual Meeting and Academic Conference for Association of IE, D357-D362, 2012.

[18] C. -H. Yu, Solving some integrals with Maple, International Journal of Research in Aeronautical and Mechanical Engineering, Vol. 1, Issue. 3, pp. 29-35, 2013.

[19] C. -H. Yu, Using Maple to study the integrals of trigonometric functions, Proceedings of the 6th IEEE/International Conference on Advanced Infocomm Technology, No. 00294, 2013.

[20] C. - -H. Yu, A study of the integrals of trigonometric functions with Maple, Proceedings of the Institute of Industrial Engineers Asian Conference 2013, Springer, Vol. 1, pp. 603-610, 2013.

[21] C.-H. Yu, Application of Maple on some type of integral problem, Proceedings of the Ubiquitous-Home Conference 2012, pp.206-210, 2012.

[22] C.-H. Yu, Application of Maple: taking two special integral problems as examples, Proceedings of the 8th International Conference on Knowledge Community, pp.803-811, 2012.

[23] T. M. Apostol, Mathematical Analysis, 2nd ed., Boston: Addison-Wesley, p269, 1975. 